\title{
Monogenoidea en Serrasalmus rhombeus (Linnaeus, 1766) de la Cuenca Amazónica Boliviana
}

\section{Monogenoidea of Serrasalmus rhombeus (Linnaeus, 1766) from the Boli- vian Amazon Basin}

\author{
Leslie Córdova ${ }^{1}$ y Antoine Pariselle ${ }^{2}$
}

\begin{abstract}
${ }^{1}$ Unidad de Limnología y Recursos Acuáticos, Universidad Mayor de San Simón, Cochabamba, Bolivia.

Email Córdova Leslie: leslie.cordova@gmail.com ${ }^{2}$ Institut de recherche pour le développement- IRD. Email Pariselle Antoine: parisell@ird.sn
\end{abstract}

Presentado: $\quad 30 / 03 / 2006$ Aceptado: $\quad 21 / 08 / 2006$

\section{Resumen}

El presente trabajo analiza la diversidad de parásitos monogenoideos como un carácter independiente, esperando que por su alta especificidad, éstos parásitos puedan caracterizar las poblaciones de sus hospederos, la piraña blanca, Serrasalmus rhombeus. El estudio se llevó acabo en cuatro sub-cuencas de la Cuenca Amazónica Boliviana (Iténez, Beni, Madre de Dios e Ichilo); se obtuvieron 1135 parásitos, los cuales fueron agrupados en seis géneros y once especies: Anacanthorus jegui, A. amazonicus, A. rondonensis, A. gravihamulatus, A. sciponophallus, Anacanthorus sp.1, Anacanthorus sp.2, Anacanthorus sp.3; Notothecium deleastum, N. deleastoideum, Notozothecium teinodendrum; Mymarothecium galeolum; Anphithecium diclonophallum y Enallothecium aegidatum. La elevada diversidad de parásitos encontrada puede deberse a que existe o existió un complejo de especies o poblaciones diferenciadas de Serrasalmus rhombeus.

Palabras clave: Monogenoideos, Serrasalmus, Cuenca Amazónica, Bolivia.

\section{Abstract}

The present work analyse monogenoids parasite diversity as one independent character, hoping they can characterize the white piranha (Serrasalmus rhombeus) populations by its high specificity. Study was carried out in four sub-basins (Iténez, Beni, Madre de Dios e Ichilo) from the Bolivian Amazon Basin. A total of 1135 parasites were obtained, belonging to six genera and 11 species: Anacanthorus jegui, A. amazonicus, A. rondonensis, A. gravihamulatus, A. sciponophallus, Anacanthorus sp.1, Anacanthorus sp.2, Anacanthorus sp.3; Notothecium deleastum, N. deleastoideum, Notozothecium teinodendrum; Mymarothecium galeolum; Anphithecium diclonophallum y Enallothecium aegidatum. The high parasite diversity could be related to one complex of populations or species of Serrasalmus rhombeus existent or extintic.

Keywords: Monogenoidea, Serrasalmus, Amazon basin, Bolivia.

\section{Introducción}

A pesar del conocimiento adquirido sobre los peces de la Amazonía boliviana, existen aún grandes vacíos de información sobre su distribución, biología, genética, sistemática y ecología (Sarmiento \& Barrera, 2003).

En Bolivia, el incremento inevitable de la población humana, podría causar un problema de sobreexplotación de peces Amazónicos (Goulding, 1981). Este es el caso de algunas especies, como el pacú Colossoma spp., en la cuenca del río Mamoré que ya muestra los primeros signos del impacto del hombre (Van Damme, 2002). Para prevenir esta disminución, se tienen dos posibilidades: iniciar una gestión sostenible de la pesca para llegar a una explotación equilibrada; y/o empezar con la piscicultura de especies nativas. Para iniciar cualquiera de estas posibilidades, antes se debe de conocer más sobre las especies y las poblaciones de peces.

Un ejemplo para empezar estos tipos de estudios es la pirańa blanca, Serralmus rhombeus (Linnaeus, 1766), que es una especie abundante y ampliamente distribuida sobre toda la Cuenca Amazónica Boliviana (Géry, 1964; Machado-Allison, 1983), por lo que esta condición la convierte en una especie modelo para el estudio de su situación poblacional.

Para investigar esta situación poblacional, y como complemento y carácter independiente a los estudios de morfología y genética, se utilizará en el presente estudio a los parásitos (Clase Monogenoidea).
De hecho, dentro de un modelo evolutivo, se considera más eficiente la especiación de los parásitos que la de los peces, porque tienen un ciclo de vida más rápido y por lo tanto se pueden encontrar mayores diferencias entre las faunas de parásitos que entre las poblaciones del huésped (Paugy et al., 1990).

Estos parásitos son bastantes específicos del huésped y muy diversos (Poulin, 2002) y parecen estar influenciados por cambios en el medio externo así como los cambios genéticos de la población del huésped (Pariselle et al., 2003).

\section{Material y métodos}

\section{Área de estudio}

El trabajo se realizó en cuatro sub cuencas de la amazonía de Bolivia que drenan hacia el alto Madera: Iténez (río San Martín), Beni (río Beni), Madre de Dios (ríos Manuripi) e Ichilo (Laguna Tambaqui) (Fig. 1).

El río Iténez o Guaporé, es uno de los afluentes más importantes del río Mamoré, es de aguas claras, la mayoría de las especies de peces registradas para este río también se distribuyen en la cuenca del Mamoré (Sarmiento \& Killen, 1998 y Navarro \& Maldonado, 2002). El río Beni se distribuye en una gran parte en los departamentos de La Paz, Beni y Cochabamba. El río Manuripi de aguas negras, nace en la República del Perú, ingresando a territorio Boliviano por las inmediaciones del Hito, en la provincia Manuripi del departamento de Pando. El río Madre de Dios, de origen de aguas blancas tiene confluencia 


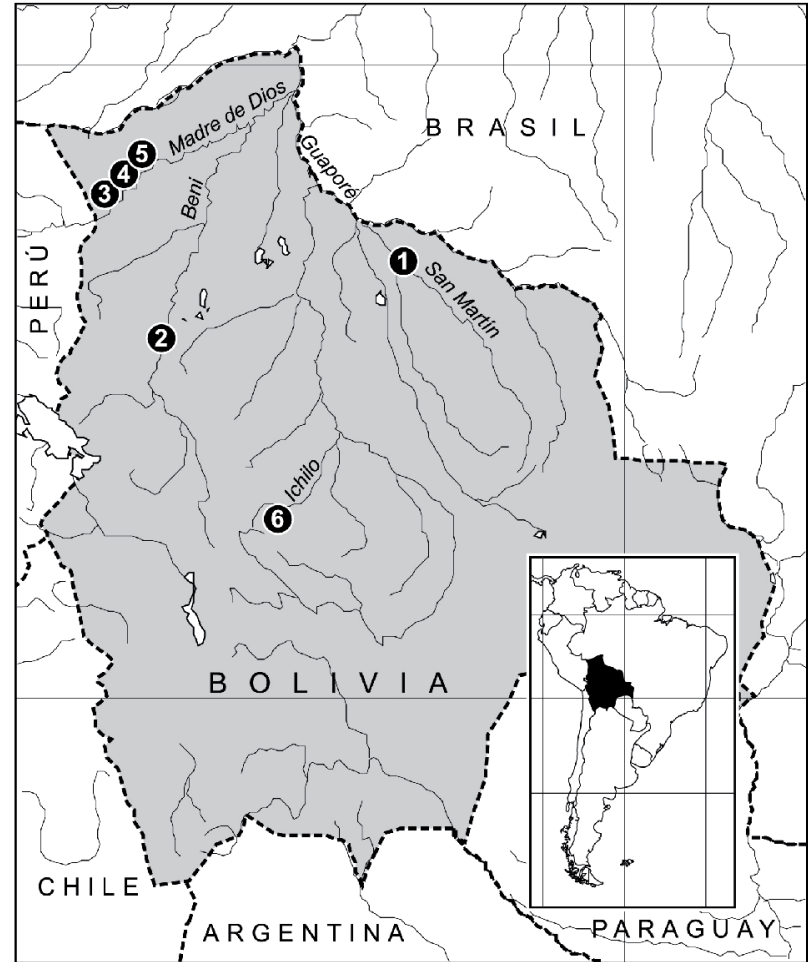

Figura 1. Ubicación de los puntos de muestreo en las cuatro cuencas de la Amazonia boliviana, en el estudio de la diversidad de monogenoideos de Serrasalmus rhombeus.

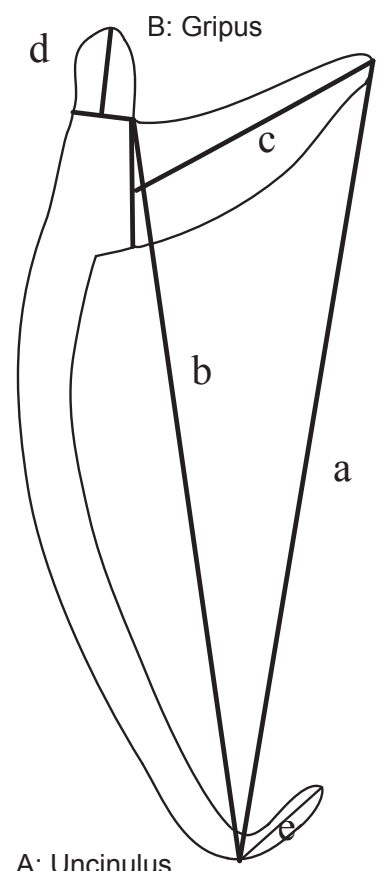

ganchos pequeños

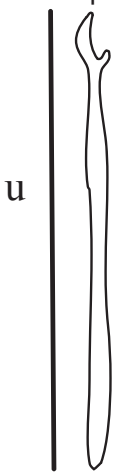

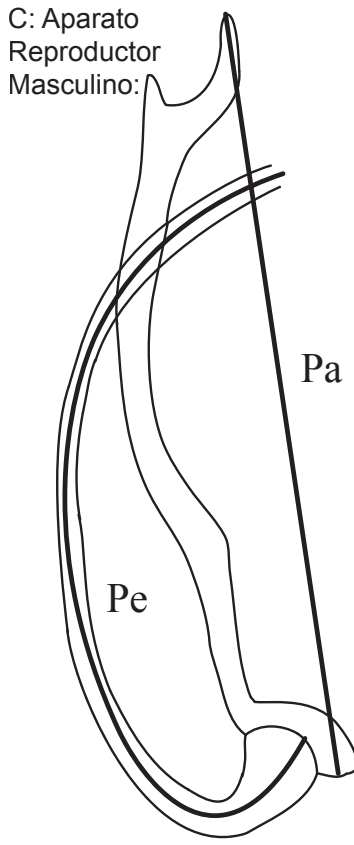

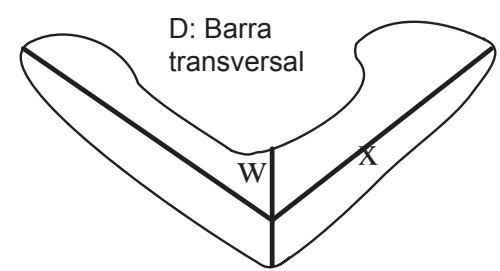

Figura 2. Formas de medición de las partes escleróticas de Monogenoidea A: Uncinulus: u (longitud de gancho pequeño); B: Gripus: a, b; c, d, e (distancias del gancho grande); C: Aparato Reproductor Masculino: Pa (longitud de la pieza accesoria), Pe (longitud del pene); D: Barra transversal: w (ancho), x (largo). con el río Beni (Navarro \& Maldonado, 2002). En este río se designaron tres puntos como Madre de Dios 1, 2 y 3, ya que se tomaron muestras dentro del mismo río y no tenían nombres específicos.

El río Ichilo esta ubicado en el centro del territorio Boliviano, abarca gran parte de la provincia Carrasco en el departamento de Cochabamba y la provincia Ichilo del departamento de Santa Cruz, limita al sur y al oeste con la cuenca del Río Grande, al oeste con la cuenca del Río Chapare. La dirección predominante que sigue es hacia el norte hasta su unión con los ríos Chapare y Grande dando origen al río Mamoré (C.E.E.D.I, 1989).

\section{Muestreo}

Se congelaron en nitrógeno líquido muestras de arcos branquiales de $S$. rhombeus, en el laboratorio se realizaron las extracciones de los parásitos monogenoideos. Una vez extraídos según la técnica de Pariselle \& Euzet, (1994) se depositaron junto a una gota de medio de Malmberg sobre un portaobjetos y se observaron en el estereo-microscopio para determinar las diferentes características.

\section{Identificación de parásitos}

La determinación de género y especie se realizó por comparación de dibujos y medidas de las partes escleróticas (ganchos dorsales/ventrales y aparato reproductor) de los parásitos colectados con material identificado disponible y descripciones publicadas (no existen claves taxonómicas para los parásitos monogenoideos en el área de estudio). La denominación y la numeración de las piezas escleróticas del haptor están definidas por Euzet \& Prost, 1981, con modificaciones de Pariselle \& Euzet (1995) para los ganchos dorsales y ventrales. Las medidas siguen la propuesta de Gussev (1962).

\section{Análisis Estadístico}

Para ver si hay diferencias entre poblaciones de parásitos (poblaciones de acuerdo a lugares o huéspedes), diferencias que resultaron de un aislamiento (y de la deriva genética), se estudió la morfometría de los parásitos con un análisis de componentes principales multivariado (ACP). Para este análisis se utilizaron 18 variables (medidas de ganchos dorsales y ventrales, ganchos pequeños y aparato reproductor del parásito) y para otros parásitos solo se utilizaron 4 variables, porque no presentaban las mismas características que los otros parásitos (ausencia de ganchos grandes) (Figura 2).

Posteriormente utilizamos un análisis de componentes factoriales $(\mathrm{ACF})$ para agrupar a los huéspedes geográficamente con presencia y ausencia de Monogenoidea. Por lo que se construyeron tablas de inventariación de los parásitos.

\section{Resultados}

Los parásitos considerados en el presente estudio son de la Clase Monogenoidea, Orden Dactylogyrinea, Suborden Dactylogyridea y Familia Dactylogyridae.

Se encontraron 1135 parásitos en 12 Serralmus rhombeus, de los cuales 343 parásitos provienen del río San Martín (3 peces), 100 del río Beni (2 peces), 210 del Madre de Dios 1 (2 peces), 78 en Madre de Dios 3 (3 peces) y del río Ichilo 401 parásitos (2 peces); en el río Madre de Dios 2 no se evidenció presencia de parásitos ( 2 peces), por lo que este río no se toma en cuenta para ningún tipo de análisis posterior. Por cada lugar se utilizaron 
Tabla 1. Especies de Monogenoidea registrados en Serrasalmus rhombeus de la Cuenca Amazónica Boliviana.

\begin{tabular}{|c|c|}
\hline Género & Especie \\
\hline \multicolumn{2}{|c|}{ Ausencia de ganchos grandes, sólo pequeños } \\
\hline Anacanthorus: & $\begin{array}{l}\text { jegui } \\
\text { amazonicus } \\
\text { rondonensis } \\
\text { gravihamulatus } \\
\text { sciponophallus } \\
\text { sp. } 1 \\
\text { sp. } 2 \\
\text { sp. } 3 \\
\end{array}$ \\
\hline \multicolumn{2}{|c|}{ Con ambos tipos de ganchos (grandes y pequeños) } \\
\hline Notothecium: & $\begin{array}{l}\text { deleastum } \\
\text { deleastoideum }\end{array}$ \\
\hline Notozothecium: & teinodendrum \\
\hline Mymarothecium: & galeolum \\
\hline Anphithecium: & diclonophallum \\
\hline Enallothecium: & aegidatum \\
\hline
\end{tabular}

dos a tres peces, y por cada pez se revisaron de uno a cinco arcos branquiales, dependiendo de la abundancia de los parásitos.

\section{Grupos de Monogenoidea}

Mediante la morfología los parásitos se pudieron separar en 5 diferentes grupos, para esta agrupación se realizó una pequeña clave taxonómica con las características más sobresalientes: presencia/ausencia y forma de ganchos grandes, longitud/forma del aparato reproductor y barras dorsales/ventrales.

A estos grupos se las clasificó en seis géneros: Anacanthorus, Notothecium, Notozothecium, Mymarothecium, Anphithecium y Enallothecium (Tabla 1).

Según el ACP se obtuvo, que los grupos se superponen entre ellos, porque hay grupos en los que existen semejanzas morfológicas muy cercanas, exceptuando el grupo 1 (Género Anacanthorus), que no se tomó en cuenta para dicho análisis por presentar claras diferencias de morfología (Figura 3). Por lo tanto este análisis muestra a 11 especies de Monogeneoidea.

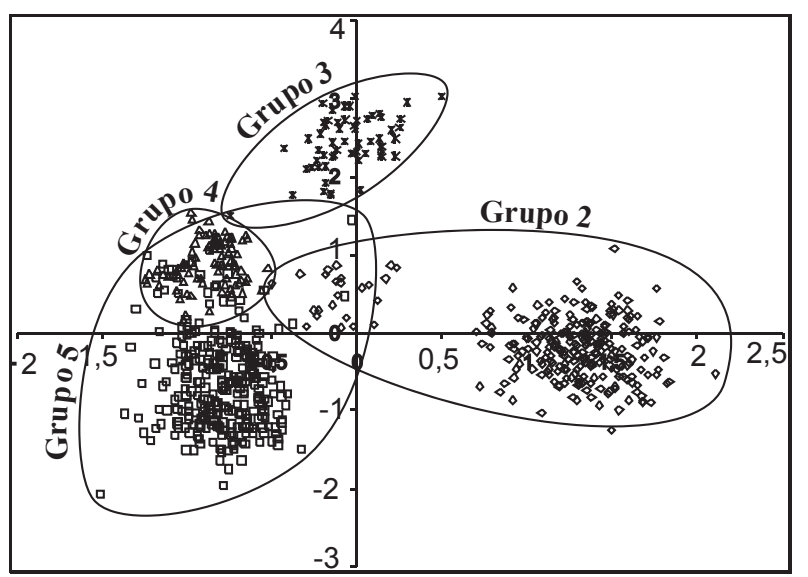

Figura 3. Análisis de Componentes Principales de los Grupos: 2 (Géneros Notothecim y Enallothecium), 3 (Género Notozothecium), 4 (Género Mymarothecium) y 5 (Género Anphithecium).

\section{Fauna parasitológica}

Como se puede observar en la tabla 2, la distribución de las especies de Monogenenoidea no es tan homogénea, existen lugares en los que se encuentran la mayoría de las especies, otros donde existen pocas especies y otros donde no hay presencia de parásitos. Esto sugiere la existencia de diferencias entre las poblaciones del huésped, posiblemente diferencias genéticas.

\section{Discusión}

\section{Abundancia y distribución de ectoparásitos}

En Bolivia es el primer estudio realizado sobre los Monogenenoidea, Kohn \& Cohen (1998) mencionan a una sola especie registrada y descrita en la Amazonía Boliviana (dentro de la elaboración de una lista de la distribución de Monogenea de peces en Sud América): Unilatus anoculus (Price, 1968) en el hospedero Hypostomus bolivianus (Loricariidae).

Nuestro trabajo revela que Serralmus rhombeus, como en otros peces hospederos de agua dulce tropicales presentan una alta diversidad de Monogenenoidea (Pariselle, 1996), ya que en el presente trabajo encontramos a seis géneros con once especies, distribuidas en cinco ríos de la cuenca amazónica de Bolivia.

La diversidad específica de parásitos puede depender de la biología del huésped y/o de la especificidad del parásito. Pariselle et al. (2003) estudiando peces continentales de la familia Cichlidae (Tilapia guineensis (Bleeker, 1862)) en África, encontraron como máximo 17 especies de Monogenenoidea, con un promedio de 6 especies por huésped y en Pangasiidae (Pangasius polyuranodon (Bleeker, 1852)) del Sudeste de Asia, un máximo de 13 especies de Monogenenoidea y un promedio de 3 especies por huésped. En el caso $S$. rhombeus se encontraron 11 especies, por lo que podemos considerarlo como bastante diverso respecto a otros huéspedes. Entonces se puede hipotetizar que por la riqueza que presenta éste, podría representar aproximadamente una media de 4 a 6 especies de parásitos por cada especie de huésped de la familia de Serrasalmidae.

La abundancia de los parásitos, es muy alta en la cuenca estudiada, más de 100 por individuos huésped, cuando son menos de 100 en los Cichlidae en África (Pariselle, 1996). No sabemos si esto es el resultado de la biología de los huéspedes (una característica de la familia Serrasalmidae) o un producto del medio ambiente (temperatura, $\mathrm{Ph}$, etc.). En el caso de las muestras del río Madre de Dios 2 no se encontraron parasitos. Tomando en cuenta que $S$. rhombeus es el mismo en los puntos 1 y 3 , se descarta la identificación del hospedero y resta como principal hipótesis de esta ausencia de parasitos, el médio ambiente.

\section{Grupos de Monogenoidea}

Según Van Every y Kritsky (1992) en S. rhombeus de la amazonia central en Brasil se registraron a 7 especies del género Anacanthorus: $A$. amazonicus, $A$. jegui, A. serrasalmi, A. gravihamulatus, $A$. mesocondylus, $A$. prodigiosus, $A$. sciponophallus. En el presente trabajo se encontraron 5 especies de las cuales solo $A$. rondonensis no esta registrada en la cuenca amazónica de Brasil y 3 especies que parecen ser nuevas y que se las denominó como Anacanthorus sp.1, sp.2 y sp.3.

Kritsky, Boeger y Jégu (1998), encontraron en la cuenca amazónica del Brasil en S. rhombeus, a Notothecim cyphophallum, $N$. deleastum, $N$. phyleticum, Enallothecium aegidatum, E. cornutum 
Tabla 2: Presencia y ausencia de las especies de Monogenoidea por río.

\begin{tabular}{|c|c|c|c|c|c|c|c|c|c|c|c|c|}
\hline \multirow{2}{*}{$\begin{array}{c}\text { Ríos } \\
\text { Especies }\end{array}$} & \multicolumn{3}{|c|}{ San Martín } & \multicolumn{2}{|c|}{ Beni } & \multicolumn{2}{|c|}{ M. Dios 1} & \multicolumn{3}{|c|}{ M. Dios 3} & \multicolumn{2}{|c|}{ Ichilo } \\
\hline & స్త & तี & స્ন & 志 & $\stackrel{10}{n}$ & 秀 & 요 & $\begin{array}{l}10 \\
\infty \\
\infty \\
\infty\end{array}$ & $\begin{array}{l}\text { ஜ } \\
\infty \\
\infty\end{array}$ & సิ & $\begin{array}{l}\text { ले } \\
\text { त्रे }\end{array}$ & 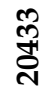 \\
\hline \multicolumn{13}{|l|}{ Grupo 1} \\
\hline Anacanthorus amazonicus & 1 & & & 2 & & & & & & & 2 & 2 \\
\hline Anacanthorus jegui & 3 & 4 & 12 & 4 & & 7 & 11 & & 1 & 3 & 16 & 5 \\
\hline Anacanthorus rondonensis & & & & & & 1 & 1 & & & & & \\
\hline Anacanthorus sciponophallus & 3 & 1 & 8 & 5 & & 1 & 2 & 1 & 3 & & 2 & \\
\hline Anacanthorus garavihamulatus & & & & & & & 1 & 1 & & & & \\
\hline Anacanthorus sp. 1 & & 3 & & & & & & & & & & \\
\hline Anacanthorus sp. 2 & & & & 1 & & & & & & & & 4 \\
\hline Anacanthorus sp. 3 & & 17 & 5 & 9 & 4 & 2 & 33 & 1 & 2 & & 16 & 7 \\
\hline \multicolumn{13}{|l|}{ Grupo 2} \\
\hline Notothecium deleastum & 23 & 35 & 11 & 19 & 3 & & 3 & & 1 & 4 & 61 & 33 \\
\hline Notothecium deleastoideum & 28 & 36 & 1 & & & & 16 & & 3 & & 40 & 24 \\
\hline Enallothecium aegidatum & 4 & 8 & & 2 & 1 & & 1 & & & & & \\
\hline \multicolumn{13}{|l|}{ Grupo 3} \\
\hline Notozothecium teinodendrum & 9 & 5 & & 7 & 3 & 3 & 7 & & & 3 & 11 & 9 \\
\hline \multicolumn{13}{|l|}{ Grupo 4} \\
\hline Mymarothecium galeolum & 16 & 6 & & & & & 23 & & 2 & & 53 & 13 \\
\hline \multicolumn{13}{|l|}{ Grupo 5} \\
\hline Amphitecium diclonophallum & 29 & 63 & 15 & 11 & 16 & 5 & 86 & 29 & 18 & 4 & 76 & 21 \\
\hline
\end{tabular}

y E. umbelliferum. En Bolivia sobre la misma especie se encontró a Notothecim deleastoideum, la cual no está presente en la cuenca amazónica del Brasil. El huésped en el que se registra a esta especie de parásito se encuentra registrada como Serrasalmus sp. $(2 \mathrm{n}=58)$ en Manaos, Enallothecium aegidatum es la única especie de este género que se encontró en $S$. rhombeus en Bolivia

Especies de los géneros Notozothecium y Mymarothecium en S. rhombeus, también de la cuenca amazónica de Brasil, fueron descritas por Kritsky, Boeger y Jégu (1996) de las cuales solo Notozothecium teinodendrum y Mymarothecium galeolum fueron registradas en este trabajo.
Para el género Anphithecium, Boeger y Kritsky (1998) registraron en $S$. rhombeus a Anphithecium diclonophallum, en la amazonía Brasilera, la misma especie que fue registrada en este trabajo.

\section{Fauna parasitologica}

La presencia de 11 especies de parásitos en $S$. rhombeus, puede ser considerada bastante abundante en comparación a otros estudios realizados sobre peces de aguas tropicales. Pariselle et al. (2003) explican este hecho como resultado de la historia y de la evolución del huésped, donde estas poblaciones se han dividido en otras diferentes poblaciones, sobre las cuales los parásitos

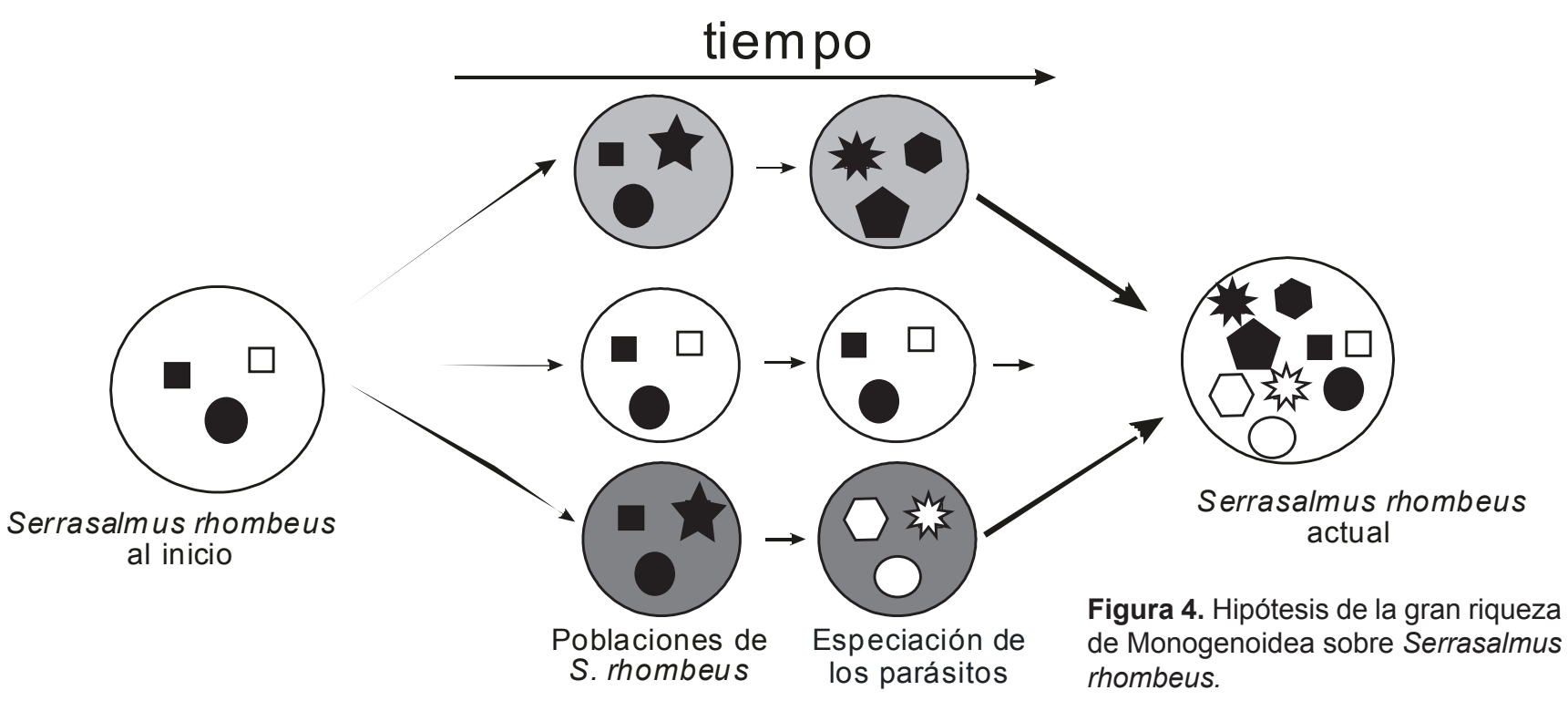


han realizado especiaciones, por lo que la riqueza en especies de parásitos va a aumentar (Figura 4).

Los resultados de ACF (Figura 5) muestran que las poblaciones de $S$. rhombeus pueden ser identificadas por la presencia y ausencia de especies de Monogenoidea, lo que no se puede hacer con la genética.

\section{Conclusiones}

Los resultados obtenidos en este trabajo, prueban que $S$. rhombeus presenta una fauna parasitológica diversa, conformada por 11 especies de parásitos y tres posibles nuevas nuevas especies.

Los parásitos de la clase Monogenoidea halladas en los cinco ríos de la cuenca amazónica de Bolivia, presentan variaciones de distribución y abundancia en comparación con otras espécies.

En el río Madre de Dios 2, no fueron encontrados parásitos, esto podría explicarse por la calidad de agua u otros factores que puede presentar el río y no asi por la biología del hospedero .

La distribución de estas especies muestra diferencias que son suficientes como para poder caracterizar poblaciones geográficas del huésped. Ya que existen especies de parásitos que se encuentran solamente en "poblaciones» del huésped, y como lo demuestran Pariselle et al. (2002), podría deberse a la existencia de un complejo de especies o de poblaciones aisladas identificadas como de $S$. rhombeus. Aún mas la diversidad de estas especies de parásitos es tan elevada entre las poblaciones de huésped, por lo que la existencia de un complejo de especies es muy probable.

El trabajo de sistemática no esta concluido, porque dentro de la sub-familia Serrasalminae, existen más o menos 20 especies, por lo que se espera que existan más de 100 especies de Monogenoidea solamente sobre esta familia de huésped en Bolivia.

Con los resultados obtenidos en este trabajo podemos ver la importancia de estudiar a los parásitos en los peces de la cuenca amazónica de Bolivia. Un mayor conocimiento de la fauna parasitológica en diferentes poblaciones de huéspedes de la Amazonía Boliviana, nos permitirá caracterizar mejor las poblaciones de peces (existencia de complejo de especies o no), teniendo un apoyo de la genética (confirmación de nuevas especies).

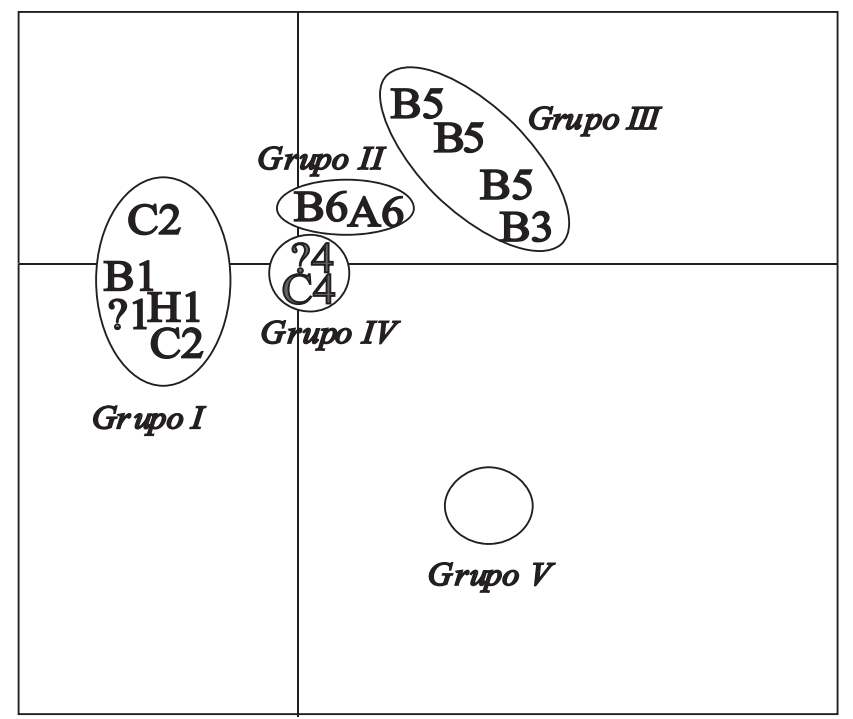

Figura 5. Análisis de componentes factoriales (ACF), de presencia y ausencia de parásitos: $A, B, C, H, ?$ = grupo genético de peces; 1, 2, 3, 4, 5 y 6 = código de localidad

\section{Literatura citada}

C.E.E.D.I. 1989. (Centro de Estudios Ecológicos y de Desarrollo Integral). Diagnostico del Estado actual de los recursos pesqueros de la cuenca Amazónica Boliviana. La PazBolivia. Pp. 143.

Euzet, L. \& M. Prost. 1981. Report of the meeting on Monogenea: problems of systematics, biology and ecology. In: Slusarski, W. (Eds) Review of advances in parasitology. Warsaw: P.W.N. Polish Scientific Publishers. pp. 1003-1004.

Géry, J. 1964. Contributions a l'étude des poissons characoïdes - 27: systématique et évolution de quelques piranhas ("Serrasalmus"). Recueil d'observations de zoologie. Dans l'ocean. Vie Milieu (Ser. C) Biol. Terr. 14(3): 597-617.

Goulding, M. 1981. Man and fisheries on an Amazon frointier. Junk Publishers. Boston-London. 136pp.

Gussev, A. 1962. In: Key to parasites of freshwater fish of the USSR. Moscow-Leningrad: Academiya Nauk. Pp 919.

Kohn, A. \& S. C. Cohen. 1998. South American Monogenea - list of species, hosts and geographical distribution. International Journal for Parasitology. 28 (1): 1517-1554.

Kritsky,D. C., W. A. Boeger. \& M. Jegú. 1998. Neotropical Monogenoidea. 31. Ancyrocephalinae (Dactylogyridae) of Piranha and Their Relatives (Teleostei, Serrasalmidae) from Brazil: Species of Notothecium Boeger and Kritsky, 1988, and Enallothecium. gen. n. Journal of the Helminthological Society of Washington. 65: 31-49.

Kritsky,D. C., W. A. Boeger \& M. Jegú. 1996. Neotropical Monogenoidea. 28. Ancyrocephalinae (Dactylogyridae) of Piranha and Their Relatives (Teleostei, Serrasalmidae) from Brazil and French Guiana: Species of Notozothecium Boeger and Kritsky, 1988, and Mymarothecium gen. n. Journal of the Helminthological Society of Washington. 63: 153-175.

Machado-Allison, A. 1983.Estudios sobre la sistemática de la subfamilia Serrasalminae (Teleostei, Characidae). Parte II. Discusión sobre la condición monofilética de la subfamilia. Acta Biológica Venezuelica. 11: 45-195.

Navarro, G. \& M. Maldonado. 2002. Geografía Ecológica de Bolivia: Vegetación y Ambientes Acuáticos. Ed. Centro de Ecología Simón I. Patiño-Departamento de Difusión. Cochabamba. Bolivia. 719 pp.

Pariselle A., S. Morand, M. Deveney \& L. Pouyaud. 2003. Parasite species richness of closely related hosts: historical scenario and "genetic" hypothesis. In: Taxonomie, écologie et évolution des métazoaires parasites. Taxonomy, ecology and evolution of metazoan parasites. (Livre hommage à Louis Euzet). Tome II. Combes C \& Jourdane J. (eds), PUP Perpignan. p. 147-166.

Pariselle, A. \& L. Euzet. 1994. Three new species of Cichlidogyrus Paperna, 1960 (Monogenea, Ancyrocephalidae) parasitic on Tylochromis jentinki (Steindachner, 1895) (Pisces, Cichlidae) in West Africa. Systematic Parasitology. 29: 229-234.

Pariselle, A. \& L. Euzet. 1995. Gill parasites of the genus Cichlidogyrus Paperna, 1960 (Monogenea, Ancyrocephalidae) from Tilapia guineensis (Bleeker, 1862), with descriptions of six new species. Systematic Parasitology. 30: 187-198.

Pariselle, A. 1996. Diversité, spéciation et évolution des Monogènes branchiaux de Cichlidae en Afrique de l'Ouest. PhD Thesis, University of Perpignan. 200 p.

Pariselle, A. Lim L.H.S. \& A. Lambert. 2002. Monogeans from pangasiidae (Siluriformes) in Southeast Asia: IV. Five new species of Thaparocleidus Jain, 1952 (Ancylodiscoididae) from Pangasius krempfi, P. Kunyit, P. Mekongensis and P. Sabahensis. Parasite. 9:315-324. 
Paugy, D., J. F. Guégan \& J. F. Agnèse. 1990. Three simultaneous and independent approaches to the characterization of a new species of Labeo (Teleostei, Cyprinidae) From West Africa. Canadian Journal of Zoology. 68: 1124-1131.

Poulin, R. 2002. The evolution of Monogenean diversity. International Journal for Parasitology. 32: 245-254.

Sarmiento, J. \& T. Killen. 1998. Hidrología de la meseta de Huanchaca y sus alrededores. In: Pillen, T. S. Schulenberg (Eds.). A biological assessment of Parque Noel Kempff Mercado, Bolivia. RAP Working Papers 10, Conversation International, Washintong D.C. p. 55-57.

Sarmiento, J. \& S. Barrera. 2003. La Riqueza de Bolivia. Estado de conocimiento y conservación. Ed. F. A. N. Santa Cruz de la Sierra - Bolivia.
Van Damme, P. 2002. Disponibilidad, sostenibilidad y calidad de los recursos hídricos en Bolivia. Cumbre Mundial sobre el Desarrollo Sostenible, Johannesburgo.

Van Every, L. R. \& D. C. Kritsky. 1992. Neotropical Monogenoidea. 18. Anacantorus Mizelle and Price, 1965 (Dactylogyridae, Anacanthorinae) of Piranha (Caracoidea, Serrsalmidae) from the Central Amazon, their phylogeny, and aspects of host-parasite coevolution. Journal of the Helminthological Society of Washington. 59: 52-75. 